Proceedings of the Operational Research Society Simulation Workshop 2021 (SW21)

M. Fakhimi, D. Robertson, and T. Boness, eds.

DOI: https://doi.org/10.36819/SW21.015

\title{
Analysing the Effect of Food Supply Chain Traceability on Product Waste
}

Mr. Rob Glew

Insitute for Manufacturing

University of Cambridge

rg522@cam.ac.uk

\section{Dr. Marco Perez Hernandez}

Institute for Manufacturing

University of Cambridge

mep53@cam.ac.uk

\author{
Prof. Duncan McFarlane \\ Institute for Manufacturing \\ University of Cambridge \\ dm114@cam.ac.uk
}

\begin{abstract}
This paper presents initial results from an agent-based simulation study into the impact of supply chain traceability information sharing on food waste reduction in the fresh food supply chain. Based on data collected during a 2019 study of a multi-tier citrus fruit supply chain, a conceptual model of information sharing relationships and their impact on food waste was created. This model formed the basis of an agent-based simulation where the actors in the supply chain share time-and-temperature storage information for the fruit. The model is successfully verified against the case study data, initial findings show that the benefits of traceability are realised further downstream in the supply chain. We further comment on the environmental and social implications of our results.
\end{abstract}

\section{Keywords:}

Supply Chain Visibility, Food Waste, Agent-based Model

\section{INTRODUCTION}

Food waste is a pressing economic, environmental, and social concern. In the UK alone, approximately 10 million tonnes of food was wasted in 2016 (WRAP 2017). Over $60 \%$ of fresh food waste could have been avoided by better practices in the supply chain and more sensible purchasing by consumers (Barratt and Oke 2007). Globally, total food waste is over 1.3 billion tonnes and is growing (Gustavsson et al. 2011). Higher income countries waste a higher proportion of total food production than lower income countries, leading to social inequality (Gustavsson et al. 2011). All told, avoidable food waste in the UK contributes as much as $3600 \mathrm{Kg}$ of $\mathrm{CO} 2$ equivalent environmental impact per tonne of waste, or approximately 36 billion Kgs of CO2 equivalent per year (Tonini et al. 2018).

Given this, there is strong political and industrial interest in interventions to reduce food waste (de Moraes et al. 2020). However, the breadth of academic studies into food waste reduction in the food supply chain (FSC) is limited, focusing primarily on perishability-related pricing practices (Grillo et al. 2017) or on consumer action (Morone et al. 2018). Recently, firms in all industries have begun to consider the sustainability of their whole supply chain, rather than solely their own activities (Villena and Gioia 2020). This has led to calls for greater supply chain traceability, transparency, and information sharing (Gardner et al. 2019).

The optimisation of a perishable food product supply chain requires information sharing to match supply with demand and to ensure products are sold according to their quality and residual shelf-life (RSL) (Spada et al. 2018).

With this simulation we seek to understand the relationship between food waste and traceability information sharing in a perishable food supply chain. We ask: What is the impact of traceability on 


\section{Glew, Perez Hernandez, and McFarlane}

food waste in a perishable food supply chain? and address the question with a quantitative, agent-based simulation of a citrus food supply chain. Empirical data obtained from a case study is used to validate the model. The use of an agent-based approach allows the model to consider the competitive impact of supply chain partners sharing information, as it is acknowledged that firms are often unwilling to share data they perceive as commercially sensitive (Bartlett et al. 2007).

\section{FOOD WASTE MODELLING APPROACHES}

This study uses the definition of traceability from Olsen and Borit (2013), "The ability to access any or all information relating to that which is under consideration, throughout its entire life cycle, by means of recorded identifications" (pp. 147). In this case, specifically regarding storage time and temperature traceability information. Following Barratt and Oke (2007) we define visibility as "the extent to which actors within a supply chain have access to or share information which they consider as key or useful to their operations" (pp. 1218) and define information sharing as an activity intended to produce or increase visibility.

Food waste is the most pressing issue in global food supply chains (Gustavsson et al. 2011). It has, therefore, attracted significant industrial and academic attention. Food waste is generally defined as food lost throughout the supply chain from farm to fork, including damage of crops during harvest, damage arising from transport, incorrectly processed or handled food, and expired food (Griffin et al. 2009).

The studies by de Moraes et al. (2020), Liljestrand (2017), and Kaipia et al. (2013), indicated that more information sharing and better IT integration would reduce food waste. Improved inventory management practices and order picking result in maximising the residual shelf life (RSL) of products in the supply chain (Kaipia et al. 2013).

The application of simulation approaches to food waste reduction practices is limited. Such studies have focused solely on the relationship between food waste, food perishability, and pricing. Authors approach the problem from the economic perspective, rather than the environmental or social sustainability perspectives. As such, the goal of these studies is to maximise profit for the retailer by reducing the amount of product they dispose of, ignoring the multi-tier perspective.

Wang and $\mathrm{Li}$ (2012) developed a simulation where pricing decisions are based on dynamically identified RSL of the food product. Quality is modelled as a dynamic state that decays linearly over time, until it passes a threshold where the product can no longer be sold. The objective is to estimate as closely as possible the quality and RSL of the products on the retailer's shelves and adapt the price to maximise sales. Yu and Nagurney (2013) adopted a similar approach, where the objective is to maximise the RSL at the retail stage.

More recent studies of the relationship between pricing strategies, perishability, and food waste include Chang et al. (2016), where the authors included an agent-based simulation of consumer preferences. Chen et al. (2019) used a linear decay rate of quality and RSL in a game theoretic simulation of a two-echelon food supply chain. Here the goal was to maximise the profits for the both the supplier and the retailer. Grillo et al. (2017) considered the relationship in the context of Spanish fruit supply chains, a similar context to the case study of this paper. Integer non-linear programming was used to maximise the RSL of products arrive with the consumer.

In a literature review of food supply chain modelling approaches, Zhu et al. (2018) concluded that food waste simulation had not been explored beyond the objective of maximising profit through pricing strategies. In pointing to future research directions, the authors highlighted the need to understand the relationship between traceability, visibility, and sustainability in food supply chains. To date, no models have addressed the value of information sharing to food waste reduction. Zhu et al. (2018) also highlighted the lack of models that account for the increasing role of digital technologies such a supply chain traceability systems in supply chain management, which enable further collaboration and coordination. As a consequence, this study sets out to address the specific issue of the potential impact traceability could have on food waste. 


\section{MODEL DEVELOPMENT}

\subsection{Case Background}

This study is based on data collected during a case study research project of a multi-tier fresh food supply chain. Supply chain data was collected and in-depth interviews were conducted with a leading fresh fruit distributor in the UK. This firm supplies a major supermarket chain, has an annual turnover of over $£ 200$ million and sources from over 500 farms in 11 countries. In their largest product line, soft citrus, over $65 \%$ is sourced from Spain, equating to 3500 truck loads annually. This modelling study focuses solely on the Spanish soft citrus supply chain for the partner firm.

This firm was selected as they had recently implemented a full chain traceability system for storage time and temperature information. Figure 1 shows a schematic representation of the actors in the supply chain. Growers (farm cooperatives who are also responsible for packing the fruit) supply a UK-based distributor via 3rd party logistics. A second transport stage takes fruit from the distributor to the retailer's locations around the UK, where they are stored before being displayed for sale to consumers.

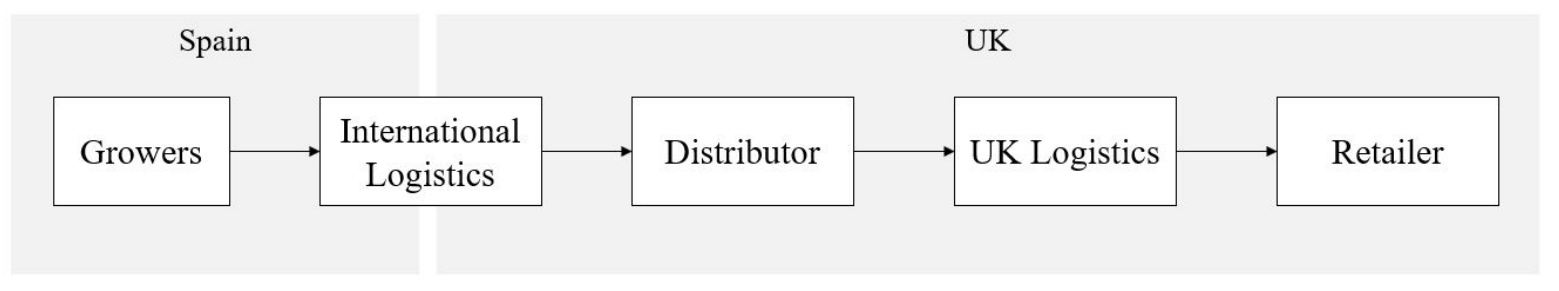

Figure 1: Schematic supply chain map

In 2015, before the traceability system was implemented, the waste level for Spanish soft citrus was $2.54 \%$. In 2019, once the traceability was in place and part of business-as-usual operations, the waste level at reduced to $1.86 \%$. In this supply chain, waste primarily occurs due to the inability of actors to accurately estimate the residual shelf life of the products. The most desirable inventory management policy is "First to Decay, First Out", however, without detailed traceability about the life of the product it can be impossible to estimate RSL. RSL is a function of product age and storage conditions (Rong et al. 2011). A smaller portion of waste occurs due to mishandling of products during transit, this is estimated to be between 10 and $15 \%$ in this case.

\subsection{Selection of Modelling Methodology}

An agent-based model was selected for this study due to its ability to simulate the dynamics and interactions between the self-interested agents in a complex system such as the supply chain (Herrera et al. 2020). Simulation is growing methodology in supply chain research and agent-based approaches have been used to study supply chain risk aversion (Cannella et al. 2019), technology adoption (Basingab 2019), and many other phenomena. In the context of this study, traceability information can be competitively sensitive and firms may be hesitant to share it with partners. This effect can be included in agent behaviour to increase the accuracy of the model.

\subsection{Key Elements of Simulation Model}

The simulation model incorporates four types of agents that represent the key actors in the soft citrus supply chain presented in Figure 1. The model focuses in the operation of the distribution echelon when dealing with different configurations of visibility of products with the aim of minimising the product wastage along the chain.

A key agent type is the Distributor agent that gathers products coming from growers and dispatches them to the retailer. The distributors receive orders from retailers and breakdown these orders to place individual orders to growers. The order periodicity can be parameterised. Once the product is received, the processing time of the distributor is also parameterised and represents the time for quality control and handling, previous to the dispatching. The distributor uses a "First to Decay, First Out" inventory management policy by default. Hence, the more visible the information about actual remaining shelf life of the product, the better the dispatch decisions the distributor makes to reduce product wastage. 


\section{Glew, Perez Hernandez, and McFarlane}

Upstream, the Grower agents encapsulates the activities between harvesting and packing of product, until the produce is ready to circulate along the chain for fulfilling the orders. The production is based on a normal distribution, whose mean is based on the production capacity and can be parameterised together with its standard deviation. The growers dispatch product following a FIFO policy, based on the orders received from the distributor. Each grower has different processing times and also different levels of information sharing. Hence, a grower might deliver full traceability information whereas other do not. On the other end of the chain, the Retailer agent incorporates the behaviour for periodically placing orders to distributor according to the demand forecasts. The demand follows a normal distribution, whose mean and standard deviation can be parameterised. Once product is received, this is offered to customer and sold within a given user-defined time.

Finally, the Transporter agents enable the flow of product along the chain. Each transporter covers a distance for delivery of products based on real-world map and route. The time it takes to deliver products depends on the parameters of velocity, the distance and the maximum number of hours that can be driven per time step.

One important feature of the model is the product decay dynamics. We adopt the methodology from Rong et al. (2011) which uses a linear decay rate dependent on the environmental temperature and the time. The decay, for a time period of length $\tau$ and a temperature $T$, is given by the equation 1 .

$$
\Delta q(\tau, T)=-\kappa_{0} \tau \cdot \exp \left[\frac{-H}{T}\right]
$$

The constants $H$ and $-\kappa_{0}$ depend on the fruit type. In the agent model, each agent has a temperature for storing the products which is applied for as long as the agent is in control of the product. Then, the product wastage along the chain depends on the temperatures at which each agent stores the product and the storage duration. If temperature were stable along the chain the decay rate would be always the same, however, the model incorporates more realistic random variability of the temperature, especially in the transporter agents, as these are the ones that are more susceptible to experience variations in the storage temperature of the product. Hence, transporters might store the product at the standard expected temperature or at a random temperature, given a user-defined parameter (more details in section 4.2).

Another key aspect of the model is the traceability information flow. This behaviour intends to replicate the dynamics of the supply chain when there is a traceability system in place that enables information sharing among the agents. We model this situation by incorporating two components that represent delays and noise in the traceability information. The delay is defined in time units and represents how long it takes for an agent to have access to RSL information after receiving the product. Likewise, the noise is a random variable that enables the simulation of information accuracy. The noise is normalised to 1 and follows a normal distribution whose standard deviation can be defined for each specific scenario.

\section{SIMULATION STUDY}

In this section we present the simulated scenarios studied with the model described in the previous section and present the main results of the study.

\subsection{Scenarios}

We are interested in the effect of information sharing in the total wastage along the chain. So we identify two key scenarios, as follows:

- Low traceability: This is the case where real-time information about temperature and storage time for each product was not available. Hence, the distributor does not have accurate nor timely information about the residual shelf-life of the product.

- High traceability: In this case, the traceability system is in place and the distributor can base its inventory management policies on complete, accurate, and updated residual shelf-life information of the product. 


\subsection{Modelling Parameters}

The agent-based model is parameterised to replicate the case study supply chain. The Spanish soft citrus season runs from 1st September to 31st July (302 days). Over this period, 7.5 million cases of fruit enter the supply chain at the growers end. Cases are transported in trucks from Spain to the UK. The desired storage temperature throughout is $3^{\circ} \mathrm{C}$. Information in the traceability system is shared in parallel to the flow of products.

There are seven agents as detailed in Table 1 . In this case there is only one single retailer and a distributor agent as we are not studying differences among these actors. However, we incorporate two growers and their corresponding international transporters to account for the differences at these stages of the chain as they provide the information inputs that the distributor uses for dispatching the product to retailer.

Table 1: Agents and Simulation Parameters.

\begin{tabular}{|l|l|l|l|}
\hline Agent & Parameter Name & Value & Units \\
\hline Grower 1 & default storage time & 2 & time steps \\
prower 2 & $\begin{array}{l}\text { production capacity } \\
\text { default storage time }\end{array}$ & 12 & product cases \\
time steps \\
production capacity & 12 & product cases \\
Transporter 1 (International) & prob temperature & 0.9 & \\
& distance & 1200 & miles \\
& velocity & 60 & miles/hour \\
Transporter 2 (International) & prob temperature & 0.5 & \\
& distance & 1200 & miles \\
& velocity & 60 & miles/hour \\
Transporter 3 (UK) & prob temperature & 0.9 & \\
& distance & 80 & miles \\
Distributor & velocity & 40 & miles/hour \\
Retailer & processing time & 1 & time steps \\
& max shelf time & 1 & time steps \\
\hline
\end{tabular}

All agents have a standard temperature parameter set to $3^{\circ} \mathrm{C}$. Grower agents have starting stock, a default storage time (representing the minimum time required for processing), and the production capacity. One time period represents a day. Transporter agents have distance (in miles) and velocity parameters (miles per hour). Transports also have a temperature probability(prob_temperature), which indicates the "reliability" of that agent in storing fruit at the desired temperature. For example, an agent with a prob_temperature $=0.9$ has the standard temperature $90 \%$ of the time and $10 \%$ of the time a randomly selected temperature between $3^{\circ} \mathrm{C}$ and $10^{\circ} \mathrm{C}$. One informant in the case study estimated that sometimes over $40 \%$ of readings from trucks can be erroneous. The distributor has a processing time parameter to cover the time required for quality control and handling. Finally, the retailer has a maximum shelf time product that represents their stock turns.

For the fruit decay model, $E_{a}$ and $-\kappa_{0}$ constants were obtained from data provided by Snart et al. (2006) with a life of 21 days at $8^{\circ} \mathrm{C}$ and 56 days at $3^{\circ} \mathrm{C}$. $\mathrm{R}$ is the universal gas constant.

$$
H=\frac{-E_{a}}{R}=15200 \quad \text { and } \quad \kappa_{0}=1.55 \times 10^{22}
$$

These parameters remain stable for the two scenarios analysed. To consider the variations between both scenarios, we used the traceability information parameters info_delay and info_noise. As we have products with two different origins (grower 1 and 2), in the Low Traceability scenario, products originated from grower 1 were set to an info_delay sufficiently high that information would not be shared until after products had decayed; for grower 2, the delay was set to 1 day. In the latter case, the standard deviation of the noise was set to 0.02, meaning that reported product RSL would randomly be $+/-2 \%$ of its actual RSL. This value was obtained after model calibration to case study 
data. For the High Traceability scenario, both parameters were set to 0 for all products to simulate perfect information sharing.

We ran the model 100 times for each scenario. A burn-in period of 200 time steps allowed for transients to decay and for the retailers and distributors to build up some starting stock.

\subsection{Model Validation}

Granular empirical data to validate every aspect of the model was not available as this information is not shared transparently within the supply chain. However, given that the simulation parameters were derived from the case study data, it is sufficient in this case to ensure that the waste levels in the model match those from the case study. The waste levels of the simulation were fine tuned to match the figures from the empirical data by adjusting the rate of consumption (or demand) in the model to ensure products flowed through the supply chain. This demand rate was not available from the case study data as the retailer considered it sensitive information.

Table 2: Validation of Results to Case Data (given assumption of constant demand)

\begin{tabular}{|l|l|l|}
\hline Data & Simulation Result & Empirical Data \\
\hline High Traceability Waste & $1.87 \%$ & $1.86 \%$ \\
Low Traceability Waste & $2.56 \%$ & $2.54 \%$ \\
\hline
\end{tabular}

\subsection{Simulation Results}

In this section we present the results from the study. Table 2 shows the that the waste levels in the simulation very closely match those of the case study, with a maximum percentage error of $0.8 \%$. In the Figures 2, 3 and 4 the time $=0$ is 200 days after the start of the simulation due to the burn-in period. The results are the average after 100 runs of the simulation. The simulation configuration and resulting data are available in GitHub ${ }^{1}$.

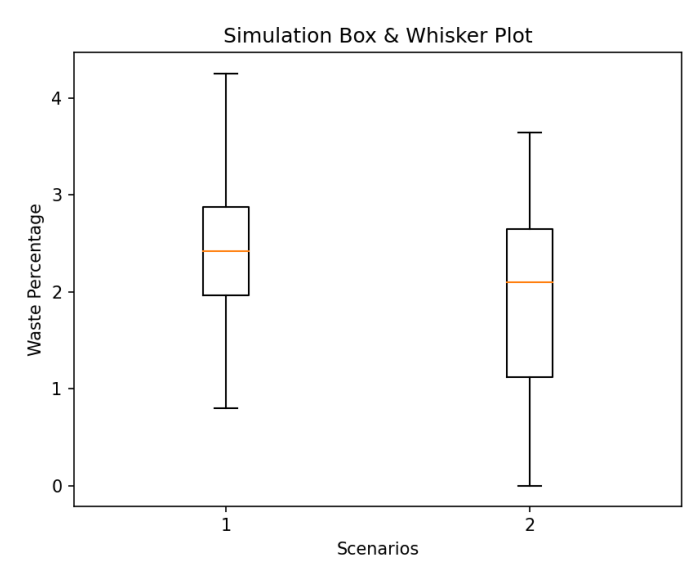

(a) Boxplot Comparison of Scenario 1 (Low Traceability) and Scenario 2 (High Traceability) - Aggregation of 100 Runs

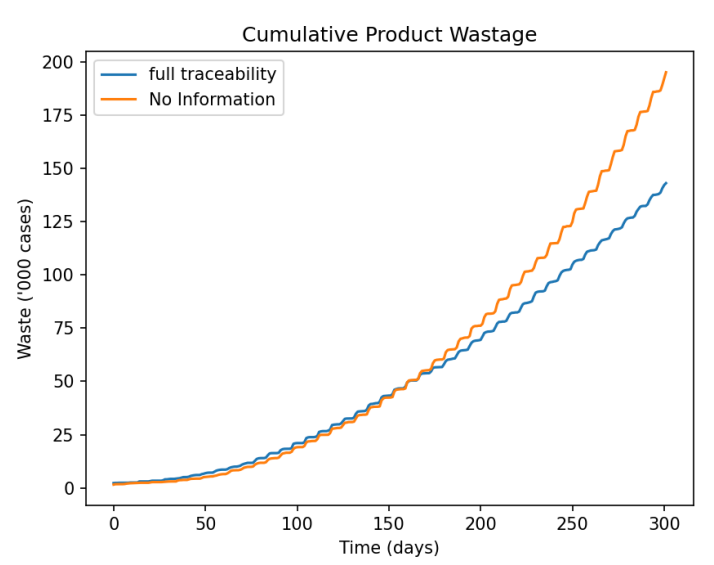

(b) Cumulative Waste

Figure 2: Cumulative Plots (all agents)

\section{DISCUSSION}

In this section of the paper, we discuss the results presented above and their implications. We begin with the main findings of the study by comparing the two scenarios. We then discuss the economic and environmental implications of these findings. Finally, we consider the limitations to this simulation.

\footnotetext{
${ }^{1}$ https://github.com/mperhez/scvis-plots/tree/master/sw21
} 


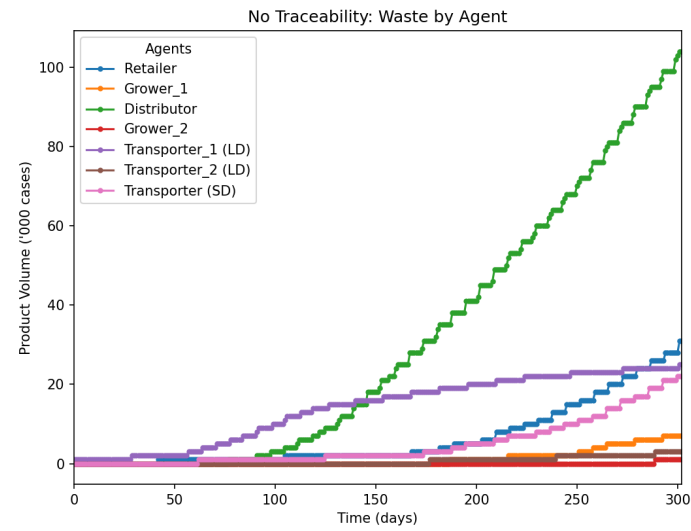

(a) Low Traceability Case

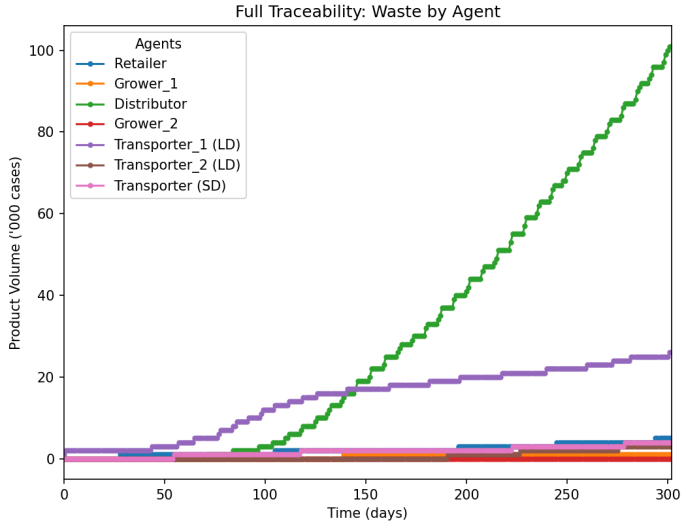

(b) High Traceability Case

Figure 3: Waste by Agent

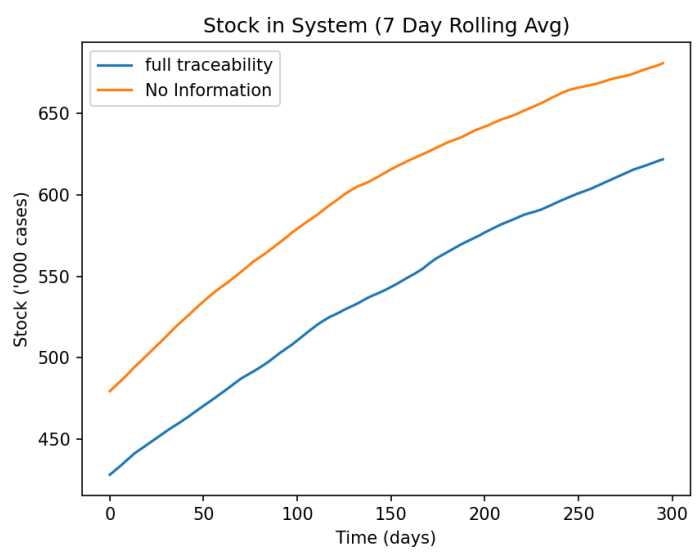

Figure 4: Stock Level in the Supply Chain

\subsection{Traceability System Analysis}

Based on the results of this simulation experiment, we develop four key insights regarding the relationship between traceability systems and food waste. First, we are able to observe where in the supply chain the food waste reductions occur when a traceability system is implemented. The reduction can be explained as the product traceability enables complete, timely and accurate information sharing along the chain, that in turns makes it possible to implement a "First to Decay, First Out" inventory management policy that has effect in the food waste within the observed scenarios.

Secondly, in figure $3 \mathrm{~b}$ we observe that the waste level in the two scenarios is closely matched in the first half of the season, then the results diverge in the second half. Figure 3 suggests that this acceleration in waste level is caused by an increase in waste at the retailer and their logistics provider (TransporterSD). Without traceability information, the error in the guessing for inventory management builds up throughout the season. The downstream end of the supply chain receives the product at its oldest and therefore its highest perishability risk. Given this, the retailer and their transporter would be expected to see the greatest reduction in food waste and this is supported by the simulation experiment.

Next, we find it interesting to consider this experiment against the body of qualitative evidence that retailers are usually the actor that forces the development of a supply chain traceability system (Aung and Chang 2014). Our simulation study provides clear evidence for the reasons behind this trend; in terms of food waste, retailers see the greatest gains from implementing the traceability system. The distributor estimates that each wasted case costs $£ 15.40$ to the retailer, so a reduction from 38,000 cases 
to 4,000 cases in a season is a cost saving of $£ 520,000$ per season for the retailer alone. Extending this cost-per-case-analysis, the results of the simulation study suggest the growers and international logistics providers will have little motivation to invest in the traceability system. The distributor sees an $8 \%$ reduction in waste, so will be somewhat more motivated that the growers to invest in the system.

Finally, when making the comparison between Figures 2 and 4, we observe that stock levels have reduced as well as waste levels. This indicates there may be relationship between stock levels and product waste. This matches expectations based on common sense. Higher stock increases the product volume that is likely to expire while in stock. More investigation is needed to understand the nature relationship between stock levels and waste at each agent in the supply chain. Further research is planned in this area.

\subsection{Economic, Environmental, and Social Impact}

Wang and Li (2006) called for more research into the "value" of supply chain traceability and data for traceability beyond the so-called "traditional" metrics. This simulation experiment indicates a number of fruitful avenues for further exploration along the triple-bottom-line perspective. Firstly, in the previous sub-section we considered the economic value of supply chain traceability in terms of cost of a wasted case. We noted that the economic benefits of traceability accrue closest to the consumers. Turning to the environmental impact of product waste, consider that product wasted at the retailer in the UK has already resulted in carbon emissions relating to its transport and storage up to that point. Therefore, the environmental impact of food waste is lower in the earlier stages of the supply chain. Traceability data could be applied to ensure that waste in the downstream supply chain is reduced, for example, by setting a residual shelf-life threshold.

Thirdly, considering social impact of product waste, fruit growing has historically been associated with poor labour practices. The production of food that eventually goes to waste could be considered to be incurring an ethical cost. This simulation study provides the insight that supply chain traceability can provide social value by reducing over-production to account for food waste and therefore the amount of labour required.

\subsection{Limitations of the Study}

This study presents several limitations that will be addressed in future works. The model does not incorporate a separate component for representing product waste due to handling. Although the case study shows this figures are low, incorporating this component will enable to study better the effect of traceability system performance.

The wastage in the system is already a very small amount that even small variations will look high, as a result the figures that we obtain, for the standard deviation of the relative waste among runs, look high. Although these values are consistent, we plan to incorporate new empirical data so the calibration of the model can be improved.

We have assumed a constant level of supply and demand throughout the season, however this is not an accurate reflection of reality. Both production of citrus fruit and demand follow cyclical patterns throughout the season. We plan to incorporate further empirical data to simulate these trends.

\section{CONCLUSIONS AND FUTURE WORK}

In this paper we have presented the development of a model to quantify the relationship between supply chain traceability information sharing and food waste. The model was trialled and validated using empirical data obtained from a European citrus fruit supply chain. We demonstrated the model was able to replicate the food waste seen in the case study data to an accuracy of better than $1 \%$. The simulation experiment proved its value by presenting four insights into supply chain traceability systems:

- Supply chain traceability systems can reduce the food waste from perishability

- The retailer and final stage logistics provider see the greatest reduction in food waste

- The retailer is likely to be most motivated to invest in supply chain traceability, whilst the growers least so, as the reduction in wastage is greater further downstream in the supply chain

- Higher stocks are associate with a higher wastage level 
We further considered how the simulation experiment provided insights into the economic, environmental, and social value of a supply chain traceability system. Finally, we point to some possible extensions to this model which the authors consider it valuable to explore:

1. Information Quality - Miller (1996) noted 10 dimensions of information quality. The simulation model could be extended to assess the impact of changes in these dimensions of quality in traceability information on the level of food waste. For example, by reducing the "completeness" of the information.

2. Further empirical validation - As noted in the discussion of the limitations of this study, the collection of more empirical data from citrus fruit supply chains would enable the validation of the model at a more granular level.

3. Application to other supply chains - A further extension of the simulation model would be the application to other produce supply chains by changing the parameterisation. For example, the length of the season, the perishability of the produce, and the locations of growers. Through this, more generalisable conclusions can be obtained.

\section{ACKNOWLEDGEMENTS}

The authors would like to thank AMT Fresh and Dr. Mukesh Kumar for their support in the case study for this project. We would also like to thank GS1 for their ongoing support in this field of research.

\section{REFERENCES}

Aung, M. M., and Y. S. Chang. 2014. "Traceability in a food supply chain: Safety and quality perspectives". Food Control 39:172 - 184.

Barratt, M., and A. Oke. 2007. "Antecedents of supply chain visibility in retail supply chains: A resource-based theory perspective". Journal of Operations Management 25:1217-1233.

Bartlett, P. A. et al. 2007, August. "Improving supply chain performance through improved visibility". The International Journal of Logistics Management 18:294-313.

Basingab, M. 2019. "Investigating the adoption of Internet of Things technology using agent-based simulation". Proceedings of the International Conference on Industrial Engineering and Operations Management 2019 (MAR): 1539-1550.

Cannella, S. et al. 2019. "An exploratory study of risk aversion in supply chain dynamics via human experiment and agent-based simulation”. International Journal of Production Research 57 (4): 985-999.

Chang, X. et al. 2016. "Agent-based simulation of pricing strategy for agri-products considering customer preference". International Journal of Production Research 54 (13): 3777-3795.

Chen, X. et al. 2019. "Optimal pricing strategy for the perishable food supply chain". International Journal of Production Research 57 (9): 2755-2768.

de Moraes, C. C. et al. 2020. "Retail food waste: mapping causes and reduction practices". Journal of Cleaner Production 256:120124.

Gardner, T. A. et al. 2019, September. "Transparency and sustainability in global commodity supply chains". World Development 121:163-177.

Griffin, M. et al. 2009, December. "An analysis of a community food waste stream". Agriculture and Human Values 26:67-81.

Grillo, H. et al. 2017. "Mathematical modelling of the order-promising process for fruit supply chains considering the perishability and subtypes of products". Applied Mathematical Modelling 49:255 -278 .

Gustavsson, J. et al. 2011. "Global food losses and foodwaste". Technical report, FAO.

Herrera, M. et al. 2020. "Multi-agent systems and complex networks: Review and applications in systems engineering". Processes 8 (3): 1-29.

Kaipia, R. et al. 2013, April. "Creating sustainable fresh food supply chains through waste reduction". International Journal of Physical Distribution and Logistics Management 43:262-276.

Liljestrand, K. 2017. "Logistics Solutions for Reducing Food Waste". International Journal of Physical Distribution \& Logistics Management 47:318-339. 
Miller, H. 1996. "THE MULTIPLE DIMENSIONS OF INFORMATION QUALITY”. Information Systems Management 13 (2): 79-82.

Morone, P. et al. 2018, June. "Does food sharing lead to food waste reduction? An experimental analysis to assess challenges and opportunities of a new consumption model". Journal of Cleaner Production 185:749-760.

Olsen, P., and M. Borit. 2013. "How to define traceability". Trends in Food Science \& Technology 29 (2): $142-150$.

Rong, A. et al. 2011. "An optimization approach for managing fresh food quality throughout the supply chain". International Journal of Production Economics 131 (1): 421 - 429. Innsbruck 2008.

Snart, J. et al. 2006. "Oranges: Safe Methods to Store, Preserve, and Enjoy". Technical report, University of California: Division of Agriculture and Natural Resources.

Spada, A. et al. 2018. "The influence of shelf life on food waste: A model-based approach by empirical market evidence". Journal of Cleaner Production 172:3410 - 3414.

Tonini, D. et al. 2018, June. "Environmental impacts of food waste: Learnings and challenges from a case study on UK". Waste Management 76:744-766.

Villena, Veronica and Gioia, Dennis 2020. "A More Sustainable Supply Chain". Available at https://hbr.org/2020/03/a-more-sustainable-supply-chain Accessed 31 August 2020.

Wang, X., and D. Li. 2006. "Value Added on Food Traceability: a Supply Chain Management Approach". In 2006 IEEE International Conference on Service Operations and Logistics, and Informatics, 493498.

Wang, X., and D. Li. 2012. "A dynamic product quality evaluation based pricing model for perishable food supply chains". Omega 40 (6): 906 - 917. Special Issue on Forecasting in Management Science.

WRAP 2017. "Estimates of Food Surplus and Waste Arisings in the UK-1 Estimates of Food Surplus and Waste Arisings in the UK". Technical report, The Waste and Resources Action Programme.

Yu, M., and A. Nagurney. 2013. "Competitive Food Supply Chain Networks with Application to Fresh Produce". European Journal of Operational Research 22:273-282.

Zhu, Z. et al. 2018. "Recent advances and opportunities in sustainable food supply chain: a modeloriented review". International Journal of Production Research 56 (17): 5700-5722.

\section{AUTHOR BIOGRAPHIES}

ROB GLEW received a MEng in manufacturing engineering and management from the University of Cambridge in 2019. He is currently a Doctoral Researcher at the Institute for Manufacturing, University of Cambridge, where his research focuses on the strategic value of traceability systems in food and medical supply chains.

MARCO PEREZ HERNANDEZ is Research Associate at the Institute for Manufacturing of the University of Cambridge. He received his PhD in Computer Science from the University of Leicester in 2018. His research interests include the use of multi agent systems and services architectures to engineer complex industrial systems.

DUNCAN MCFARLANE is Professor of Industrial Information Engineering at the Cambridge University Engineering Department, and head of the Distributed Information \& Automation Laboratory within the Institute for Manufacturing. He has been involved in the design and operation of industrial automation and information systems for twenty years. 